\title{
Cardiac Resynchronisation Therapy in Heart Failure Patients with Atrial Fibrillation
}

\author{
Maurizio Gasparini, Alessio Cappelleri, Paola Galimberti, Carlo Ceriotti and Angelica Montorio
}

IRCCS Istituto Clinico Humanitas, Rozzano/Milano

\begin{abstract}
Cardiac resynchronisation therapy (CRT) is an important device-based, non-pharmacological approach that has been shown to improve left ventricular (LV) function and reduce both morbidity and mortality rates in selected patients affected by advanced heart failure (HF), with New York Heart Association (NYHA) functional class III-IV, ejection fraction (EF) $\leq 35 \%$, QRS duration $\geq 120 \mathrm{~ms}$ and on optimal medical therapy. Patients with atrial fibrillation (AF), who constitute an important subgroup of HF patients, are nowadays considered eligible for receiving CRT as described in the latest European Society of Cardiology (ESC) and American Heart Association/American College of Cardiology/Heart Rhythm Society (AHA/ACC/HRS) guidelines, with some relevant differences in terms of how to manage the interference of natural rhythm and biventricular pacing. In this article, the authors explain how AF may interfere with adequate CRT delivery and how to manage different AF burdens, trying to obtain the best effects of CRT in AF patients.
\end{abstract}

\section{Keywords}

Atrial fibrillation, heart failure, resynchronisation, defibrillators, ablation

Disclosure: The authors have no conflicts of interest to declare.

Received: 23 April 2010 Accepted: 17 May 2010 citation: European Cardiology, 2010;6(2):92-5

Correspondence: Maurizio Gasparini, IRCCS Istituto Clinico Humanitas, Via Manzoni 56 Rozzano-Milan, Italy. E: maurizio.gasparini@humanitas.it

Cardiac resynchronisation therapy (CRT) is an important device-based, non-pharmacological approach that has been shown to improve the outcome in selected patients with chronic heart failure (HF). Large randomised trials have clearly demonstrated that CRT improves left ventricular (LV) function and reduces both morbidity and mortality rates. ${ }^{1-5}$ Until a few years ago, CRT was indicated in patients with advanced HF (New York Heart Association [NYHA] functional class III-IV), reduced LV systolic function (ejection fraction $[E F] \geq 35 \%$ ), evidence of electrical dyssynchrony (determined by QRS duration $\geq 120 \mathrm{~ms}$ ), on optimal medical treatment and who were in sinus rhythm (SR). ${ }^{6}$ In the latest European Society of Cardiology (ESC) and American Heart Association/ American College of Cardiology/Heart Rhythm Society (AHA/ACC/HRS) guidelines, ${ }^{7,8}$ atrial fibrillation (AF) patients, who constitute an important subgroup of HF patients, ${ }^{9}$ have been considered eligible to receive CRT on the condition that the effects of underlying rhythm are neutralised by atrio-ventricular junction (AVJ) ablation. Despite that clear statement, current guidelines do not bring clearly defined approaches to the different forms of AF (paroxysmal, persistent and permanent). The aim of this article is first, to explain how AF interferes with adequate CRT; second, to provide a brief overview on the effects of CRT in AF patients, followed by some recommendations based on current clinical evidence on the most adequate approach in terms of AF form, with special emphasis on the extent of atrial arrhythmic (AT/AF) burden; and lastly, to examine how new evidence concerning SR resumption after $\mathrm{CRT}$ in patients affected by permanent $\mathrm{AF}$ is reported.

It is important to point out that for the time being these recommendations remain unsupported by randomised controlled trials (RCTS), which are obviously much needed. ${ }^{10}$

\section{Atrial Fibrillation Rhythm Interferes with Adequate Delivery of Cardiac Resynchronisation Therapy}

AF (whether paroxysmal, persistent, or permanent) poses a number of challenges for adequate CRT delivery. An intrinsic, intermediate to high, irregular spontaneous AF rhythm reduces the percentage of effectively biventricular paced (BVP) captured beats percentage. Even in a patient who has normal AF rate, phases of effective biventricular capture will alternate with phases of competing higher AF rhythm that causes spontaneous, fusion or pseudo-fusion beats (see Figure 1). This suggests that the effective global 'CRT dose' may be markedly reduced in comparison with atrial-synchronous rhythm and a short AV interval (as is achieved during normal SR), as the number of effective biventricular captured beats may be strongly reduced. Moreover, in AF patients, during exertion spontaneous ventricular rate tends to over-ride biventricular (BIV) pacing rates, thus determining a further reduction of paced beats precisely when patients are most in need of having correct and constant BIV capture. This kind of behaviour is expected to greatly limit functional capacity.

Another problem is the possible negative impact on prognosis of combined use of negative chronotropic drugs in order to achieve adequate rate control. In fact, some studies 'indirectly' suggested that the use of either digoxin or amiodarone in HF patients may increase morbidity and mortality. ${ }^{4}$

Adequate management of AF and other atrial arrhythmias is primarily based on defining the AT/AF burden and its negative impact on CRT delivery. 


\section{Defining Atrial Arrhythmic Burden in Heart Failure Patients Treated with Cardiac Resynchronisation Therapy}

Defining atrial arrhythmia burden is derived from an integration of clinical, device-derived data, as well as instrumental findings (such as echocardiographic measures). Any HF patient with a history of atrial arrhythmias requires particular care, especially in the first months after CRT, in order to ensure that cardiac resynchronisation is adequately delivered.

From a clinical standpoint, it is important to identify symptoms such as palpitations and, more importantly, the worsening of effort dyspnoea, which may suggest that the resynchronisation effect is reduced because of the interference of the underlying irregular atrial rhythm. These clinical observations should be substantiated by instrumental data (for instance echocardiographic data), which may show an unchanged status or further progression of LV dysfunction (for example increased ventricular volumes, increased filling pressures and further LVEF reduction). Retrieving relevant information (such as BVP percentage, duration and numbers of 'mode switch' episodes, etc.) through device monitoring (see Figure 2) is an additional important factor: its implementation to the abovementioned complement of clinical and echocardiographic data may provide a complete picture of the extent of AF/AT burden in a single patient. These different aspects, all available during a routine outpatient visit, allow a correct assessment of the effective AF/AT burden influencing CRT delivery. The AT/AF burden may be classified as high, intermediate or low. Recently, Steinberg et al. ${ }^{11}$ pointed out the importance of an accurate evaluation of CRT dose using sophisticated 12-lead Holter monitoring, which seems to be more accurate than conventional device-based information, in order to better quantify pure BIV pacing beats compared with fusion or pseudofusion beats.

\section{Medical Therapeutic and Device-based Options for 'Rate Control' in Low Atrial Fibrillation Burden}

The rate-control strategy encompasses treatment options that effectively reduce and regularise the heart rate in patients who usually have permanent or persistent AF and who cannot be readily cardioverted to SR. Claims of rate-control drugs and/or activation of device-based algorithms are reasonable as a first-line approach when AF/AT burden is low/intermediate.

Rate-control drugs considered effective in HF patients with depressed LV function include digoxin, amiodarone and betablockers. However, more recent findings derived from randomised trials have suggested caution in the use of digoxin and amiodarone in patients with $\mathrm{HF} .^{12}$

Some device-derived features may be helpful in improving rate control and thus ameliorating CRT delivery. These features include ventricular rate regularisation (VRR), which performs BIV pacing by overriding intrinsic rhythm through faster ventricular-paced depolarisation, allowing the reduction of short RR cycles through retrograde concealed penetration of the AV node..$^{13,14}$ The benefits of rate control achieved by VRR activation have been demonstrated by acute haemodynamic benefits in patients with permanent AF and patients with only mild HF treatment and single chamber right ventricular (RV) pacing. ${ }^{15}$ Another useful feature is the 'ventricular
Figure 1: Atrial Fibrillation Interferes with Correct Biventricular Stimulation

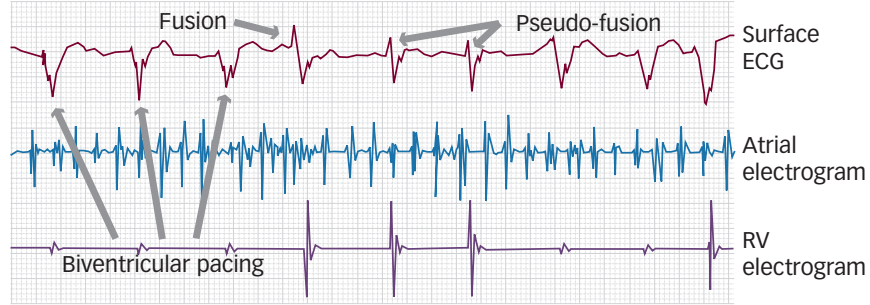

In a patient with atrial fibrillation (AF) and heart failure treated with cardiac resynchronisation therapy (CRT), spontaneous irregular intrinsic beats alternate with fusion and pseudo-fusion beats, thus markedly reducing effective CRT. As shown in this figure, this may occur even during normal rate $A F$. ECG = electrocardiography; $R V=$ right ventricular.

Figure 2: Different Aspects of Cardiac Resynchronisation Therapy Before and After Atrioventricular Junction Ablation

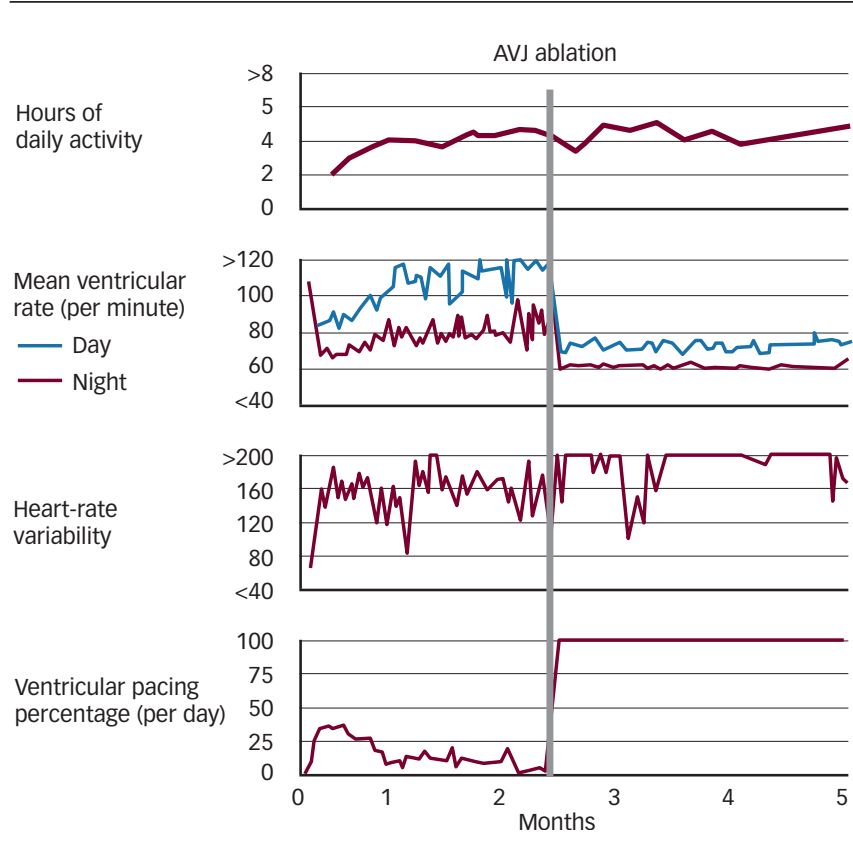

This figure shows different aspects of cardiac resynchronisation therapy (CRT) patients with atrial fibrillation (AF) before and after atrio-ventricular junction (AVJ) ablation, which may be appreciated through device features. In a female 59 years of age with permanent AF treated with a CRT and defibrillator (CRT-D). AVJ ablation yielded the following improvements: better functional status as shown by the number of hours of activity per day, maximisation of biventricular pacing percentage and improvement of heart-rate variability profile.

sense response' (also called 'trigger' function), which triggers LV pacing after premature-RV-sensed event detection. ${ }^{16}$ In the context of CRT, the effectiveness of these algorithms combined with the use of rate-control drugs has not been investigated in randomised controlled trials, while data derived from large observational cohort studies have yielded contrasting results. ${ }^{15-17}$ One of these studies ${ }^{17}$ in particular provided evidence that the administration of negative chronotropic drugs combined with device algorithm activation (VRR and trigger mode), even if allowing $85 \%$ of BIV stimulation, did not yield significant long-term improvements in functional status, LVEF increase and endsystolic volume reduction. The ineffectiveness of this approach was confirmed by another more extensive multicentre European study, which reported a higher mortality rate, particularly due to worsening progressive HF in AF patients treated with negative chronotropic drugs. ${ }^{18}$ By contrast, other smaller studies ${ }^{19-21}$ have stated that in order to achieve positive results after CRT in terms of improved survival, an aggressive rate-control strategy is not necessary. However, it is worth 
Figure 3: Comparison of Kaplan-Meier Analysis for Freedom from Death by Any Cause Between Four Studies

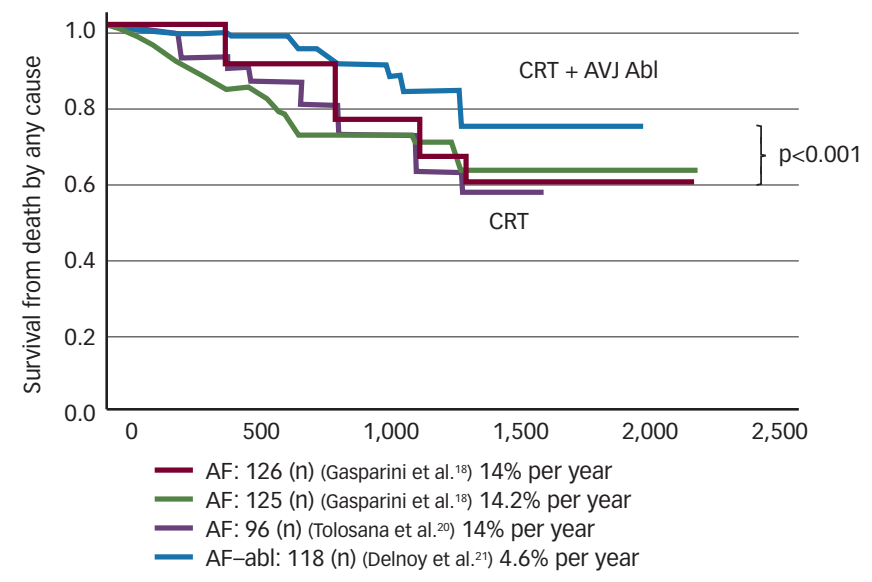

The figure is adapted from (red) Gasparini et al., ${ }^{18}$ (green) Khadjooi et al., ${ }^{19}$ (purple) Tolosana et al. ${ }^{20}$ and (blue) Delnoy et al. ${ }^{27} \mathrm{AF}=$ atrial fibrillation; $\mathrm{AF}$-drugs = atrial fibrillation with preserved atrio-ventricular (AV) node conduction; AF-abl = atrial fibrillation group with ablated $A V$ node; $S R=$ sinus rhythm.

emphasising that when the survival curves of the HF patients treated with a combined device-based/drug regimen are compared ${ }_{1}^{22}$ the total mortality rate was remarkably high, amounting to $>14 \%$ per year of both separate cohorts of non-ablated patients (see Figure 3). Therefore, it follows that in HF patients treated with CRT who present a high or intermediate AT/AF burden, the pursuit of an aggressive treatment strategy, such as AVJ ablation, is warranted.

\section{Atrioventricular Junction Ablation for the Management of Atrial Fibrillation in Heart Failure Patients Treated with Cardiac Resynchronisation Therapy}

Until recently, AVJ ablation in AF patients treated with CRT has mainly been confined to selected patients in whom high-rate AF or atrial tachycardia jeopardises satisfactory BIV stimulation (BVP\%), and also to CRT-implantable cardioverter defibrillator (ICD) recipients receiving inappropriate ICD interventions. ${ }^{23}$ The problem of inappropriate ICD discharges during fast AF, constituting approximately $30 \%$ of all ICD interventions ${ }^{23}$ with an important negative impact on the quality of life of patients, may be completely and definitively solved by AVJ ablation. However, in the context of CRT in HF patients with concomitant $A F$, there is a growing amount of evidence supporting the usefulness of AVJ ablation. This relatively simple procedure may be useful to optimise CRT delivery by eliminating the deleterious haemodynamic effects of a competing, irregular and spontaneous intrinsic rhythm. The MUltisite STimulation In Cardiomyopathies (MUSTIC) AF randomised tria ${ }^{24}$ was the first randomised trial demonstrating possible benefits of CRT in HF patients with permanent AF and conventional indications for CRT, showing that in these patients the preferred mode of ventricular stimulation was BIV compared with RV. The study enrolled AF patients with either slow-rate AF or patients who had undergone previous ablation of the AV node; the effects between pacing modes were compared using a cross-over design with two three-month periods. While no difference was found in the intention-to-treat analysis between the two modes (due to the high numbers of drop-outs), HF patients who per protocol completed the study significantly improved in terms of functional status ${ }^{24}$ with BIV pacing. The majority of patient $\mathrm{s}^{25}$ maintained these effects for one year.
Two other prospective studies investigated the effects of pacing mode in the management of AF with rapid ventricular rates following AVJ ablation. The Optimal Pacing SITE (OPSITE) trial ${ }^{26}$ showed that rate control achieved by AVJ ablation significantly improved symptoms and functional status, while no difference between the two pacing modalities, whether LV or RV, was detected. However, the study population presented significantly less compromised LV function at enrolment. The Left Ventricular-Based Cardiac Stimulation Post AV Nodal Ablation Evaluation (PAVE) ${ }^{27}$ trial further confirmed the benefits of the 'ablate and pace' approach using different pacing modes. The latter study observed greater benefits with BIV pacing mode in patients with depressed LV EF (considered as $\leq 45 \%$ ) and/or in NYHA functional class III.

Further observational studies analysed the acute and short-term effects of AVJ ablation in HF patients with AF who were treated with CRT. These patients demonstrated an increase in global LV function, mitral regurgitation reduction and an increase in exercise capacity. ${ }^{28-30}$ Other studies confirmed the chronic effects of CRT in this patient subgroup and reported improvements in NYHA class, exercise capacity and global LV function. ${ }^{17,24,25,31,32}$ It is important to stress that these benefits appear to be confined to AF patients with previous AVJ ablation or spontaneous low-rate AF.

One large observational prospective investigation ${ }^{17}$ specifically evaluated the effects of AVJ ablation on CRT delivery using a predefined protocol. This study showed that only those AF patients who underwent AVJ ablation (and thus approaching 100\% effective BIV pacing) showed significant improvements in LVEF, LV end-systolic volume definition (LVESV) and exercise capacity. Furthermore, a significantly higher proportion of responders (responders defined as patients with $a \geq 10 \%$ reduction in LVESV) was observed in the AVJ ablation group (68\%) compared with the non-ablated group (18\%) at 12 months. Subsequently, a more extensive observational multicentre study performed by the same group, revealed that CRT combined with AVJ ablation conferred a significant reduction of deaths by any cause compared with CRT alone, particularly by reducing deaths by progressive HF.

Current observational data on AF populations treated with CRT shows that the benefits of AVJ ablation in allowing appropriate CRT delivery seems to outweigh the risks associated with pacemaker dependency creation. The peculiarity of CRT devices (using both an RV and LV pacing lead for ventricular stimulation) should, theoretically, reduce the risks of pacemaker dependency related to lead fractures or malfunction, even more by using bipolar leads. Nonetheless, the fear of pacemaker dependency remains a limiting aspect for the wider spread of AVJ ablation.

\section{Sinus Rhythm Resumption After Cardiac Resynchronisation Therapy in Patients with Permanent Atrial Fibrillation}

CRT may positively affect atrial electrophysiology (especially by lessening the stretch and change in local and systemic hormonal state). Analyses of small retrospective data sets suggest the possibility that CRT may contribute to the maintenance of SR. ${ }^{33}$ Reviewed AF trend data in CRT, as measured by the implanted device, found a reduction in AF burden after a few months of CRT. Moreover, when successfully treated with CRT, some patients with long-standing or permanent AF exhibit a surprising resumption of spontaneous SR (SRR) 
despite one or more years of continuous AF. However, only a few anecdotal case reports of SR resumption (SRR) of permanent AF after CRT have been described. ${ }^{34}$

A recent large multicentre, retrospective analysis by Gasparini et al. ${ }^{35}$ demonstrated an approximate $10 \%$ rate of spontaneous (i.e. without cardioversion or anti-arrhythmic drug) return of durable SR, usually within six months of implantation.

The factors that predicted the return of SR included smaller left atrial dimension, smaller LV dimension, shorter QRS duration during CRT and treatment by AVJ ablation. The first two factors suggest better pre-CRT characteristics but cannot be controlled. A shorter QRS suggests less residual dyssynchrony during CRT, but limitations of current LV lead implantation techniques and the inherent restricted opportunities imposed by patient venous anatomy make this difficult to control. The co-existence of three predictors versus none to two predictors increases the likelihood of SRR 3.5-fold, while the presence of all four factors improves the probability by a factor of 5.7. Critically, the subgroup of those who resumed SR went on to have a superb prognosis and a much lower death rate than those who remained in AF (0 versus 18 per 100 person-years). These data may demonstrate an ameliorated prognosis and an increase in survival rate in patients resuming SR after CRT (and AVJ ablation). It may also indicate a need to implant an atrial lead in AF patients undergoing CRT, especially those with three or four of the above-mentioned characteristics.

\section{The Need for Randomised, Controlled Clinical Trials}

There are two ongoing trials aiming to investigate the benefits of adding AVJ ablation to CRT in HF patients with AF: the Atrio-VEntricular Junction Ablation Followed by Resynchronization Therapy in Patients With Congestive Heart Failure and Atrial Fibrillation ${ }^{36}$ and the AV Node Ablation in Cardiac Resynchronisation Therapy (An-Art) study. ${ }^{37}$ They are both concerned with understanding how AVJ ablation coupled with BIV pacing may significantly improve functional capacity compared with pharmacological therapy in HF patients with permanent AF and depressed EF. Data from these two trials, together with the previous large non-randomised experience, ${ }^{17,18}$ could better clarify the usefulness of the different strategies in AF patients with HF treated with CRT.

\section{Conclusions}

The AF burden in HF patients has a significant negative impact on the clinical benefit of CRT. AVJ ablation is a fundamental weapon at the disposal of physicians: it helps to achieve full CRT delivery, enabling marked improvements in global cardiac function and in overall survival. However, it is of paramount importance for the adjustment of diagnostic and therapeutic strategies to utilise sophisticated and precise instruments to define the degree of AF burden. Only with tailored treatment approaches, can any single AF patient may be correctly treated with CRT in order to attain the best results from this important non-pharmacological treatment.
1. Cazeau S, Leclercq C, Lavergne T, et al., N Engl J Med, 2001;344:873-80.

2. Abraham WT, Fisher WG, Smith AL, et al., N Eng/ I Med, 2002;346:1845-53.

3. Auricchio A, Stellbrink C, Sack S, et al., J Am Col Cardiol, 2002;39:2026-33.

4. Bristow MR, Saxon LA, Boehmer J, et al., N Engl J Med, 2004:350:2140-50.

5. Cleland JGF, Daubert JC, Erdmann E, et al., N Eng/ J Med, 2005:352:1539-49.

6. Swedberg K, Cleland JGF, Dargie $\mathrm{H}$, et al., Eur Heart J, 2005;26:1115-40.

7. Vardas PE, Auricchio A, Blanc JJ, et al., Eur Heart J, 2007;28:2256-95.

8. Epstein AE, DiMarco JP, Ellenbogen KA, et al., Circulation, 2008;117(21): e350-408.

9. Auricchio A, Metra M, Gasparini M, et al., Am J Cardiol, 2007;99:232-8.

10. Steinberg JS, J Am Col Cardiol, 2006;48:744-6.

11. Kamath GS, Cotiga D, Koneru JN, et al., J Am Coll Cardiol, 2009;53:1050-55.

12. Bardy GH, Lee KL, Mark DB, et al., N Eng/ J Med, 2005;352:225-37.

13. Lau CP, Jiang ZY, Tang MO, Pacing Clin Electrophysiol, 1998;21:542-8.
14. Simpson CS, Yee R, Lee JK, et al., Am Heart J, 2001;142:294-300

15. Kerr AJ, Williams MJA, Stewart RAH, Am J Cardiol, 2001;87:1116-19.

16. Ciaramitaro $C$, Sgarito G, Solimene F, et al., Pacing Clin Electrophysiol, 2006;29: 866-74.

17. Gasparini M, Auricchio A, Regoli F, et al., J Am Col Cardiol, 2006:48:734-43.

18. Gasparini M, Auricchio A, Metra M, et al., Eur Heart J, 2008:29:1644-52

19. Khadjooi K, Foley PW, Chalil S, et al., Heart, 2008;94:879-83.

20. Tolosana JM, Hernandez Madrid A, Brugada J et al., Am . Cardiol, 2008;102(4):444-9.

21. Delnoy PP, Ottervanger JP, Luttikhuis $\mathrm{HO}$, et al., Am J Cardiol, 2007:99(9):1252-7.

22. Gasparini M, Regoli F, Heart, 2009;95(1):83, author reply 83-4.

23. Sweeney MO, Wathen MS, Volosin K, et al., Circulation, 2005;111:2898-2905

24. Leclercq C, Walker S, Linde C, et al., Eur Heart J, 2002;23:1780-87.

25. Linde C, Leclercq C, Rex S, et al., J Am Col Cardiol, 2002;40:111-18

26. Brignole M, Gammage M, Puggioni E, et al., Eur Heart J,
2005;26:712-22

27. Doshi RN, Daoud EG, Fellows C, et al. . J Cardiovasc Electrophysiol, 2005; 16:1160-65.

28. Garrigue S, Bordachar P, Reuter $S$, et al., Cardiac Electrophysiol Review, 2003;7:315-24.

29. Puggioni E, Brignole M, Gammage $M$, et al., J Am Col Cardiol, 2004;43:234-8.

30. Hay I, Melenovsky V, Fetics BJ, et al., Circulation, 2004;110: 3404-10.

31. Leon AR, Greenberg JM, Kanuru N, et al., J Am Col Cardiol, 2002;39:1258-63.

32. Molhoek SG, Bax JJ, Bleeker GB, et al., Am I Cardiol, 2004;94:1506-9

33. Hügl B, Bruns HJ, Unterberg-Buchwald C, et al., J Cardiovasc Electrophysiol, 2006;17(8):813-17.

34. Kies P, Leclercq C, Bleeker GB, et al., Heart, 2006;92: 490-94.

35. Gasparini M, Steinberg JS, Arshad A, et al., Eur Heart J, 2010;31(8):976-83

36. Hamdan MH, Freedman RA, Gilbert EM, et al., Pacing Clinl Electrophysiol, 2006:29:1081-8.

37. Sticherling $C$, Available at: uww. clinicaltrial.gov/ct2/results?term=Atrioventricular + $\% 28 \mathrm{AV} \% 29+$ node+ablation+in+cardiac+resynchronizatio n+therapy 